\title{
Numerical Implementation of Isotropic Consolidation of Clayey Soils
}

\author{
T. Janda, P. Kuklík, M. Šejnoha
}

This paper reports on implementation of several numerical techniques to solve a set of governing equations resulting from simple one dimensional isotropic consolidation of soils that behave according to the Cam clay model. Three different methods of solving the equations of consolidation, namely the collocation method, the finite volume method and the finite element method, are presented. Apart from evaluating their efficiency, which becomes particularly crucial when implementing these techniques in the framework of an optimization problem aimed at tuning the model parameters, a set of parameters of a Cam clay model driving the time dependent response of the soils (deformation dependent variation of the coefficient of permeability and preconsolidation pressure) is also discussed.

Keywords: Cam clay, coefficient of permeability, consolidation, finite volume method, void ratio, genetic algorithm.

\section{Introduction}

Owing to its relative simplicity, isotropic consolidation is often viewed as a basic tool for inferring the material parameters of various constitutive models. While the respective constitutive model is usually well defined and tested for the description of a certain type of soil, the values of the associated material parameters are mostly lacking, leading to an extensive laboratory program to determine them. On certain occasions, however, this problem can be confined to a single laboratory test combined with numerical simulations. In particular, this paper advocates the use of a simple isotropic consolidation test to infer the basic material parameters describing critical state models such as the modified Cam clay model.

Among others, the modified Cam clay model has been often the choice for constitutive models for a realistic representation of the inelastic behavior of clayey soils, particularly when the deformation of the solid phase is of the main concern. Its selection has been promoted by the ability of the model (with only minor modifications) to capture a number of important phenomena associated with the soil-water interaction. With reference to consolidation, the need was long ago highlighted for at least a bilinear form of the consolidation line to account for the prior loading history (unloading from a certain level of preconsolidation pressure prior to subsequent loading), which significantly affects the shape of the pressure dissipation curve.

Successful implementation of the model further requires a deformation dependent formulation of the coefficient of permeability. When expressed as a function of the actual void ratio this can significantly improve the material response, particularly when the degree of consolidation increases [8]. See also [4, 9] and Section 4 for further discussion. The above requirements then give rise to five basic material parameters to feed into the constitutive model. These parameters are summarized in Section 2, which briefly reviews the theoretical formulation of isotropic consolidation.

Manual tuning of these parameters is usually not very efficient, and should be combined with a suitable and reliable optimization technique to determine their values [10]. Here the efficiency of the selected numerical technique for solving the governing equations is of paramount importance. Therefore, a critical evaluation of the efficiency of these methods, discussed in Section 3, is one of the goals of this paper.

\section{Governing equations}

Referring to the experimental measurements carried out in the triaxial apparatus, isotropic consolidation can be viewed as a one phase ow in a fully saturated deforming medium undergoing small deformations. Neglecting the body forces, the hydrostatic state of stress maintained during the experimental measurement gives

$\sigma_{x}(x, y, z, t)=\sigma_{y}(x, y, z, t)=\sigma_{z}(x, y, z, t)=\sigma_{m}(t)$,

where $\sigma_{m}$ is the total mean stress. Following the Terzaghi-Fillunger concept of effective stresses this quantity can be expressed in terms of the pore pressure $p^{s}$ and the effective stresses between grains $\sigma_{m}^{\text {eff }}$ as

$$
\sigma_{m}=\sigma_{m}^{\text {eff }}-p^{s} \text {. }
$$

Assuming full saturation $\left(S_{w}=1,[6]\right)$ the pore pressure $p$ equals the pressure in the liquid phase $p^{w}$. Referring to the experimental conditions, the total mean stress remains constant throughout the consolidation process. The assumed stress homogeneity together with Eq. (2) then provides

$$
\dot{\sigma}_{m}=\dot{\sigma}_{m}^{\text {eff }}-\dot{p}^{w}=0
$$

where (') represents the time derivative $\partial() / \partial t$.

Transport of the liquid phase throughout the soil sample can be described by the following set of equations:

Transport equation

$$
J^{w}=-\frac{K \rho^{w}}{\gamma^{w}} \operatorname{grad} p^{w}
$$

where $J^{w}$ is the mass flux of pore water, $\gamma^{w}=g \rho^{w}$ is the specific weight of water, $\rho^{w}$ is the intrinsic mass density, and $K$ represents an instantaneous coefficient of permeability.

Balance equation reads

$$
\rho^{w} \dot{\epsilon}_{v}+\operatorname{div} J^{w}=0 \text {. }
$$

The volumetric strain $\epsilon_{v}$ follows from the

Constitutive equation

$$
\begin{array}{ll}
\epsilon_{v}=\frac{e(t)-\bar{e}_{0}}{1+\bar{e}_{0}}=-\frac{\kappa}{1+\bar{e}_{0}} \ln \left(-\sigma_{m}^{\mathrm{eff}}(t)\right), & \sigma_{m}^{\mathrm{eff}}>\bar{\sigma}_{m}^{\mathrm{eff}} \\
\epsilon_{v}=\frac{e(t)-e_{0}}{1+e_{0}}=-\frac{\lambda}{1+e_{0}} \ln \left(-\sigma_{m}^{\mathrm{eff}}(t)\right), & \sigma_{m}^{\mathrm{eff}}<\bar{\sigma}_{m}^{\mathrm{eff}}
\end{array}
$$




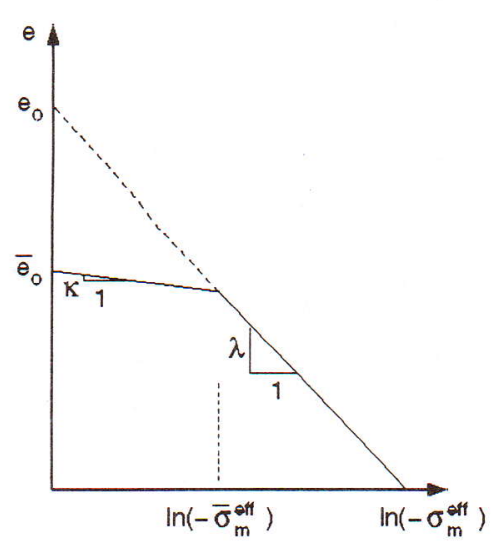

Fig. 1: Bilinear form of the consolidation line

derived in the case of the modified Cam clay model from the bilinear consolidation line, Fig. 1. The initial branch, often referred to as the $\kappa$ - line, gives evidence of the previous stress history and represents the effect of overconsolidation. The slope discontinuity between the $\mathrm{k}$ and $\lambda$ - lines can be identified with the structural strength of soil given in terms of a certain level of the effective mean stress $\bar{\sigma}_{m}^{\text {eff }}$ (preconsolidation pressure).

Differentiating Eq. (7) with respect to time gives the rate of volumetric strain in the form

$$
\dot{\epsilon}_{v}(t)=\frac{\dot{e}(t)}{1+e_{0}}=-\frac{\lambda}{1+e_{0}} \frac{\dot{p}^{w}(t)}{\sigma_{m}^{\mathrm{eff}}(t)} .
$$

Substituting Eqs. (4) and (8) into Eq. (5) and taking into account the actual triaxial set-up, in which only the bottom face of the cylinder is drained, leads to

$$
-\frac{1+e_{0}}{\gamma^{w} \lambda} \sigma_{m}^{\text {eff }}(t) \frac{\partial}{\partial z}\left(K(t) \frac{\partial p^{w}(t)}{\partial z}\right)-\dot{p}^{w}(t)=0 .
$$

It has been verified experimentally that in the case of isotropic consolidation a simple power law written as [8]

$$
\frac{K(t)}{K_{0}}=\left(\frac{e(t)}{e_{0}}\right)^{m}
$$

represents the soil behavior fairly well. The dependence of the actual void ratio $e$ on the effective mean stress, Eq. (7), together with Eq. (10) provides

$$
\frac{\partial K(t)}{\partial z}=\frac{m K(t) \lambda}{e(t) \sigma_{m}^{\text {eff }}(t)} \frac{\partial p^{w}(t)}{\partial z} .
$$

Introducing Eq. (11) into Eq. (9) finally yields

$$
\begin{aligned}
\dot{p}^{w}(t) & =-\frac{K(t)\left(1+e_{0}\right)}{\gamma^{w} \lambda} . \\
& {\left[-\frac{m \lambda}{e(t)}\left(\frac{\partial p^{w}(t)}{\partial z}\right)^{2}+\sigma_{m}^{\text {eff }}(t) \frac{\partial^{2} p^{w}(t)}{\partial z^{2}}\right] . }
\end{aligned}
$$

A similar equation can be derived for the unloading branch when replacing $\lambda$ by $\kappa$ and $e_{0}$ by $\bar{e}_{0}$ in Eq. (12)

$$
\begin{aligned}
\dot{p}^{w}(t) & =-\frac{K(t)\left(1+\bar{e}_{0}\right)}{\gamma^{w} \kappa} . \\
& {\left[-\frac{m \kappa}{e(t)}\left(\frac{\partial p^{w}(t)}{\partial z}\right)^{2}+\sigma_{m}^{\text {eff }}(t) \frac{\partial^{2} p^{w}(t)}{\partial z^{2}}\right] . }
\end{aligned}
$$

\section{Numerical implementation}

The purpose of this section is to evaluate several numerical methods commonly used when solving the consolidation problem. As mentioned above, the main objective remains computational efficiency of individual numerical techniques. First, we recall the collocation method successfully implemented in [8]. The implicit finite control volume scheme combined with the finite difference method is explored next [6]. Finally, our attention is given to the finite element method, which has proved to be an efficient tool particularly when taking the volume changes of the porous skeleton into account $[2,9]$.

Before proceeding with the present formulation of the individual numerical techniques, we recall a well known drawback of standard formulation attributed to the point of slope discontinuity along the bilinear consolidation line. This phenomenon, evident from Fig. 2 (see also [7]), results in a typical

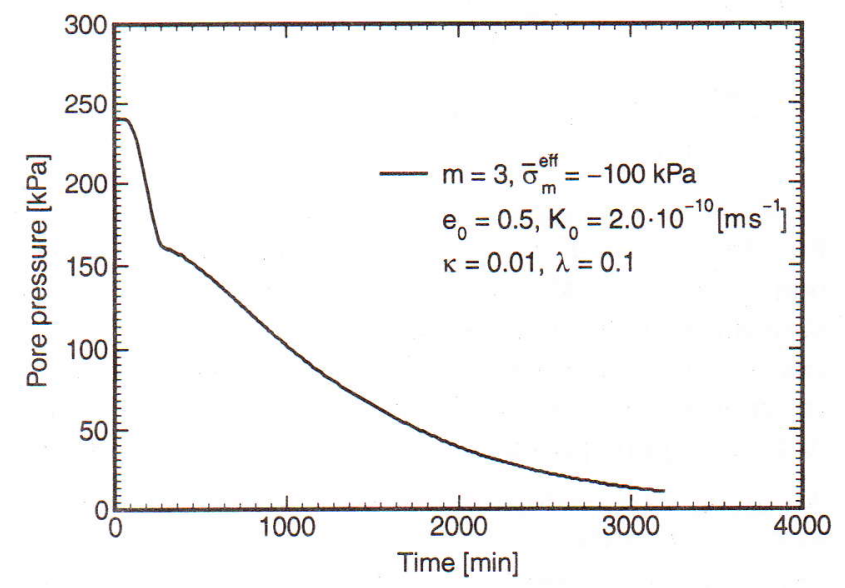

Fig. 2: Time variation of pore pressure with no smoothing of the bilinear consolidation line

double $S$ type of curve where individual segments of this curve are linked to a given slope of the bilinear consolidation line. This, however, is in contradiction with actual experimental measurements.

To avoid numerical difficulties around the point of discontinuity we equipped the original diagram in Fig. 1 with a cubic parabola to smooth out the transition zone. The numerical integration of Eqs. (12) and (13) is then carried out under certain simplifying assumptions. In particular, we first introduce an instantaneous modulus $\hat{\lambda}$ with corresponding initial void ratio $\hat{e}_{0}$ displayed in Fig. 3. These parameters, evaluated at the middle of the current time step, are then introduced in Eqs. (12) and (13), thus replacing the original variables $\kappa, \lambda$, $e_{0}$ and $\bar{e}_{0}$, respectively. Furthermore, the pore pressure dependent material parameters $K$ and $e$ are derived from an initial prediction of pore pressure taken at the end of the current time step. Details are given in Sections 3.1-3.3. 


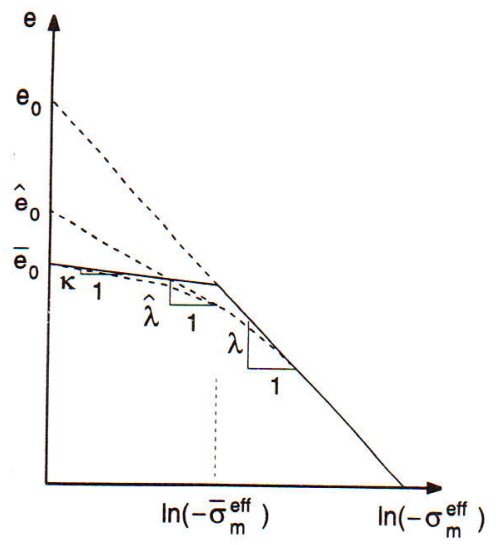

Fig. 3: Bilinear form of the consolidation line with the definition of an instantaneous modulus $\hat{\lambda}$

\subsection{Numerical solution using the collocation method}

A suitable method for solving Eq. (12) combines the collocation method along with a cubic spline approximation of the pore pressure distribution. After discretization and introduction of instantaneous parameters $\hat{\lambda}$ and $\hat{e}_{0}$ Eq. (12) becomes

$$
\begin{aligned}
\dot{p}^{w}\left(t_{j+1}\right)=-\frac{\widetilde{K}\left(t_{j+1}\right)\left(1+\hat{e}_{0}\left(t_{j+\Delta t / 2}\right)\right)}{\gamma^{w} \hat{\lambda}\left(t_{j+\Delta t / 2}\right)} . \\
\quad\left[-\frac{m \hat{\lambda}\left(t_{j+\Delta t / 2}\right)}{\tilde{e}\left(t_{j+1}\right)}\left(Y_{i}\left(t_{j}\right)\right)^{2}+\sigma_{m, i}^{\mathrm{eff}}\left(t_{j}\right) M_{i}\left(t_{j+1}\right)\right] .
\end{aligned}
$$

Note that by using the above approximation we force Eq. (12) to be fulfilled only at a certain points of collocation $(i) ; Y_{i}\left(t_{j}\right)$ and $M_{i}\left(t_{j}\right)$ then represent the first and second derivatives of the unknown pore pressure at the $i^{\text {th }}$ point, respectively. Also note that the pore pressure dependent material parameters $K$ and $e$ are found from an initial prediction of pore pressure $\widetilde{p}_{i}{ }^{w}\left(t_{j+1}\right)$ at time $t_{j+1}$, Eq. (17).

Accepting such assumptions allows the above equation to be written in the form

$\dot{p}_{i}^{w}\left(t_{j+1}\right)+\alpha\left(t_{j+1}\right) M_{i}\left(t_{j+1}\right)=\beta\left(t_{j+1}\right)$

where

$\alpha_{i}\left(t_{j+1}\right)=\frac{\widetilde{K}\left(t_{j+1}\right)\left(1+\hat{e}_{0}\left(t_{j+\Delta t / 2}\right)\right)}{\gamma^{w} \hat{\lambda}\left(t_{j+\Delta t / 2}\right)} \sigma_{m, i}^{\mathrm{eff}}\left(t_{j}\right)$,

$\beta_{i}\left(t_{j+1}\right)=\frac{\widetilde{K}\left(t_{j+1}\right)\left(1+\hat{e}_{0}\left(t_{j+\Delta t / 2}\right)\right)}{\gamma^{w} \hat{\lambda}\left(t_{j+\Delta t / 2}\right)} \frac{m \hat{\lambda}\left(t_{j+\Delta t / 2}\right)}{\widetilde{e}\left(t_{j+1}\right)}\left(Y_{i}\left(t_{j}\right)\right)^{2}$,

$\sigma_{m, i}^{\mathrm{eff}}\left(t_{j+1}\right)=\sigma_{m, i}^{\mathrm{eff}}\left(t_{j}\right)+p_{i}^{w}\left(t_{j+1}\right)-p_{i}^{w}\left(t_{j}\right)$.

To solve Eq. (15) we further assume the generalized trapezoidal rule [5] and write

$\dot{p}_{i}^{w}\left(t_{j+1}\right)=\frac{1}{\tau \Delta t}\left(p_{i}^{w}\left(t_{j+1}\right)-\widetilde{p}_{i}^{w}\left(t_{j+1}\right)\right)$,

$\widetilde{p}_{i}^{w}\left(t_{j+1}\right)=p_{i}^{w}\left(t_{j+1}\right)+(1-\tau) \Delta t \dot{p}_{i}^{w}\left(t_{j+1}\right)$,

where $t=\left(t-t_{j}\right) / \Delta t ; \Delta t=t_{j+1}-t_{j}$ is the time step. Parameter $\tau$ should be chosen from $\tau \in(1 / 2,1)$ to ensure numerical stability. The most common choice is $\tau=1 / 2$. When substituting Eqs. (16) and (17) into Eq. (15) we arrive at

$$
\begin{aligned}
p_{i}^{w}\left(t_{j+1}\right)= & -\tau \Delta t \alpha_{i}\left(t_{j+1}\right) M_{i}\left(t_{j+1}\right)+\tau \Delta t \beta_{i}\left(t_{j+1}\right)+ \\
& +\widetilde{p}_{i}^{w}\left(t_{j+1}\right) .
\end{aligned}
$$

Further suppose an equidistant spread of the collocation points with step $\Delta \mathrm{h}=\mathrm{z}_{\mathrm{i}}-\mathrm{z}_{\mathrm{i}-1}$. The cubic spline equation then reads

$\frac{1}{2} M_{i-1}+2 M_{i}+\frac{1}{2} M_{i+1}=\frac{3}{\Delta h^{2}}\left(p_{i-1}-2 p_{i}+p_{i+1}\right)$.

Combining Eqs. (19) and (18) then readily provides the final tridiagonal system of algebraic equations in the form

$$
\begin{aligned}
& {\left[\frac{1}{2}+3 \tau \Delta t \alpha_{i-1}\left(t_{j+1}\right) \frac{1}{\Delta h^{2}}\right] M_{i-1}\left(t_{j+1}\right)+} \\
& +\left[2-6 \tau \Delta t \alpha_{i}\left(t_{j+1}\right) \frac{1}{\Delta h^{2}}\right] M_{i}\left(t_{j+1}\right)+ \\
& +\left[\frac{1}{2}+3 \tau \Delta t \alpha_{i+1}\left(t_{j+1}\right) \frac{1}{\Delta h^{2}}\right] M_{i+1}\left(t_{j+1}\right)= \\
& =\frac{3}{\Delta h^{2}}\left[P_{i-1}\left(t_{j}\right)-2 P_{i}\left(t_{j}\right)+P_{i+1}\left(t_{j}\right)\right],
\end{aligned}
$$

where

$$
\begin{aligned}
P_{i}\left(t_{j}\right) & =\tau \Delta t \beta_{i}\left(t_{j}\right)+{p_{i}}^{w}\left(t_{j+1}\right) \\
Y_{i}\left(t_{j}\right) & =\frac{1}{\Delta h}\left(p_{i}^{w}\left(t_{j}\right)-p_{i-1}^{w}\left(t_{j}\right)\right)+ \\
& +\frac{\Delta h}{6}\left(2 M_{i}\left(t_{j}\right)+M_{i-1}\left(t_{j}\right)\right) .
\end{aligned}
$$

To complete the numerical procedure the following boundary and initial conditions are supplemented in accordance with the experimental set up
$p_{1}\left(t_{j}\right)=0$,
$p_{n}\left(t_{j}\right)=0$,
$p_{i}(0)=-\sigma_{m}+\sigma_{m,}^{e f f}$
${ }_{i}(0) \quad i=2, \ldots, n-1$,

where $n$ is the number of collocation points. The selected cubic spline boundary conditions assume the form

$$
M_{1}\left(t_{j}\right)=M_{n}\left(t_{j}\right)=0 \text {. }
$$

\subsection{Numerical solution using the finite volume method}

It is evident that a direct solution of Eq. (12) leads, in general, to a nonlinear system of equations. Therefore it appears preferable to start by discretizing the governing equations, which are then successively used in a single time step. In view of the one-dimensional problem, Eq. (9), the set of governing equations will be discretized using a three-layer model. The individual layers (finite control volumes) have different thickness, and diverse materials can be assigned to each layer. The pore pressure is assumed to be constant inside each control volume and within each time step, and to be equal to the pore pressure at the so called grid-point, Fig. 4. Its position is prescribed by a chosen parameter $f_{i}$.

\section{Transfer equation}

Application of the finite difference scheme converts Eq. (4) for the water flux throughout a layer $i$ with thickness $\Delta z_{i}$ into the form 


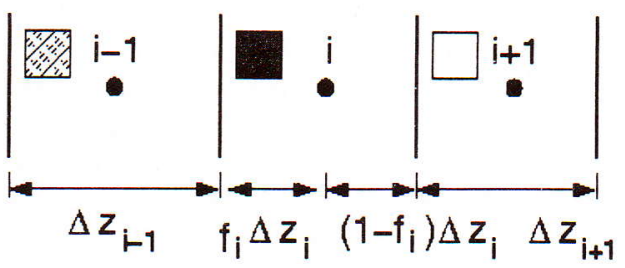

Fig. 4: A three layer model

$J_{i}^{w}=-\frac{\widetilde{K}_{i}}{g \Delta z_{i}}\left(p_{i}^{w}-p_{i-1}^{w}\right)=-\left(h z_{i}\right)\left(p_{i}^{w}-p_{i-1}^{w}\right)$,

where

$\frac{1}{h z_{i}}=\left(1-f_{i-1}\right) \frac{g \Delta z_{i-1}}{\widetilde{K}_{i-1}}+f_{i} \frac{g \Delta z_{i}}{\widetilde{K}_{i}}$,

is the overall resistance between grid points $(i-1)$ and $i$.

\section{Balance equation}

Employing the generalized trapezoidal method provides the time variation and the rate of volumetric strain in the form, recall Eqs. (16) and (17),

$\dot{\epsilon}_{v, i}\left(t_{j+1}\right)=\frac{1}{\tau \Delta t}\left(\epsilon_{v, i}\left(t_{j+1}\right)-\widetilde{\epsilon}_{v, i}\left(t_{j+1}\right)\right)$,

$\widetilde{\epsilon}_{v, i}\left(t_{j+1}\right)=\epsilon_{v, i}\left(t_{j}\right)+(1-\tau) \Delta t \dot{\epsilon}_{v, i}\left(t_{j}\right)$.

Next, applying again the finite difference scheme yields an incremental form of the balance equation (5) for layer $i$

$$
\begin{gathered}
\frac{\Delta z_{i} \rho^{w}}{\tau \Delta t}\left(\epsilon_{v, i}\left(t_{j+1}\right)-\widetilde{\epsilon}_{v, i}\left(t_{j+1}\right)\right)+ \\
+J_{i+1}^{w}\left(t_{j+1}\right)-J_{i}^{w}\left(t_{j+1}\right)=0 .
\end{gathered}
$$

\section{State equation}

Introducing Eqs. (16) and (27) into Eq. (8) gives

$$
\begin{aligned}
& \epsilon_{v, i}\left(t_{j+1}\right)- \widetilde{\epsilon}_{v, i}\left(t_{j+1}\right)=-\frac{\hat{\lambda}\left(t_{j+\Delta t 2}\right)}{1+\hat{e}_{0}\left(t_{j+\Delta t, 2}\right)} . \\
& \cdot \frac{1}{\sigma_{m, i}^{\mathrm{eff}}\left(t_{j}\right)}\left(p_{i}^{w}\left(t_{j+1}\right)-\widetilde{p}_{i}^{\omega}\left(t_{j+1}\right)\right) .
\end{aligned}
$$

After combining Eqs. (30), (29) and (25) we arrive at a set of tridiagonal algebraic equations in the form

$$
\begin{aligned}
& \left(h z_{i+1}\right) p_{i+1}^{w}\left(t_{j+1}\right)-\left[\left(h z_{i+1}\right)+\left(h z_{i}\right)+\right. \\
& \left.-\frac{\Delta z_{i} \rho^{w}}{\tau \Delta} \cdot \frac{\hat{\lambda}\left(t_{j+\Delta t / 2}\right)}{1+\hat{e}_{0}\left(t_{j+\Delta t / 2}\right)} \frac{1}{\sigma_{m, i}^{\text {eff }}\left(t_{j}\right)}\right] p_{i}^{i \prime \prime}\left(t_{j+1}\right)+ \\
& +\left(h z_{i}\right) p_{i-1}^{w}\left(t_{j+1}\right)= \\
& =\frac{\Delta z_{i} \rho^{w}}{\tau \Delta t} \frac{\hat{\lambda}\left(t_{j+\Delta t / 2}\right)}{1+\hat{e}_{0}\left(t_{j+\Delta t / 2}\right)} \frac{1}{\sigma_{m, i}^{\text {eff }}\left(t_{j}\right)} \widetilde{p}_{i}^{w}\left(t_{j+1}\right) .
\end{aligned}
$$

At the onset of consolidation the initial condition, similar to Eq. (23), reads

$p_{i}(0)=-\sigma_{m}+\sigma_{m, i}^{\mathrm{eff}}(0) \quad i=1, \ldots, n$,

where $n$ is the number of finite control volumes.

\subsection{Numerical solution using the finite element method}

In view of the general solution of fluid-solid interaction we recall the finite element method (FEM) often used to solve differential equations similar to Eq. (9). Apart from Eq. (9), which must be satisfied at any point inside the soil body, the boundary value problem requires formulation of the boundary conditions. To that end, the boundary is decomposed into two parts, $\Gamma=\Gamma_{p}+\Gamma_{q}$, with the following boundary conditions:

Essential boundary condition

$$
p^{w}=\bar{p}^{w}, \quad \text { on } \Gamma_{p},
$$

Natural boundary condition

$$
\frac{1+\hat{e}_{0}}{\hat{\lambda}} \frac{\sigma_{m}^{\mathrm{eff}}}{\gamma_{w}} K \frac{\partial p^{w}}{\partial z}+\bar{q}=0, \quad \text { on } \Gamma_{q},
$$

where $\bar{q}$ represents the prescribed fluxes. Fig. 5 shows the boundary conditions pertinent to isotropic consolidation. In this particular case, no fluid is transferred across the interface $\Gamma_{q}$ and therefore $\bar{q}=0$ and also $\frac{\partial p^{w}}{\partial z}=0$.

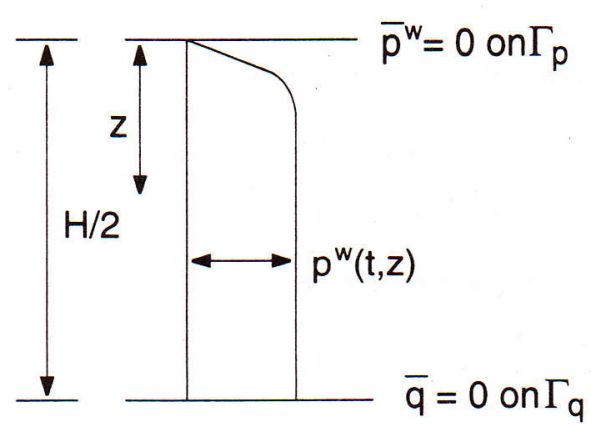

Fig. 5: Boundary conditions

To derive a set of finite element equations we start by introducing the balance equation (9) and its natural boundary condition (34) into the principle of virtual work, written as

$$
\begin{aligned}
& \int_{0}^{H}\left[-\frac{1+\hat{e}_{0}}{\gamma_{w} \hat{\lambda}_{m}^{\text {eff }}} \frac{\partial}{\partial z}\left(K \frac{\partial p^{w}}{\partial z}\right)-\dot{p}^{w}\right] \delta p^{w} \mathrm{~d} z+ \\
& +\left[\frac{1+\hat{e}_{0}}{\hat{\lambda}} \frac{\sigma_{m}^{\text {eff }}}{\gamma_{w}} K \frac{\partial p}{\partial z}+\bar{q}\right]_{z=\frac{H}{2}} \delta p^{w}(H)=0 .
\end{aligned}
$$

Integrating the first term in Eq. (35) by parts yields

$$
\int_{0}^{H} \frac{\partial \delta p^{w}}{\partial z} \frac{1+\hat{\ell}_{0}}{\gamma^{w} \hat{\lambda}} \mathrm{K} \sigma_{m}^{\text {eff }} \frac{\partial p^{w}}{\partial z} \mathrm{~d} z-\int_{0}^{H} \delta p^{w} \dot{p}^{w} \mathrm{~d} z+\bar{q} \delta p^{w}(H)=0 .
$$

The next step employs the usual approximation of the water pore pressure in the form

$$
p^{w}=\boldsymbol{N}_{p} \boldsymbol{d}_{p},
$$

where $N_{p}$ stores the element shape functions and $d_{p}$ is the vector of nodal pore water pressures. After substituting the 
above approximation into Eq. (36) we identify the following matrices, [6],

permeability matrix

$\mathbf{P}=\int_{0}^{H} \frac{1+\hat{e}_{0}}{\gamma^{w} \hat{\lambda}} K \sigma_{m}^{\text {eff }}\left(\mathbf{N}_{p}^{\prime}\right)^{\mathrm{T}} \mathbf{N}_{p}^{\prime} \mathrm{d} z, \quad N_{k, p}^{\prime}=\frac{\partial N_{k, p}}{\partial z}$,

compressibility matrix

$$
\mathbf{C}=-\int_{0}^{H} \mathbf{N}_{p}^{\mathrm{T}} \mathbf{N}_{p} \mathrm{~d} z .
$$

Note that the last term in Eq. (36) drops out due to the prescribed boundary conditions. Since Eq. (36) must be satisfied for any admissible virtual pore pressure $\delta p^{w}$ we arrive at the following system of equations

$$
\mathbf{C} \dot{d}_{p}\left(t_{j+1}\right)+\widetilde{\mathbf{P}}\left(t_{j+1}\right) d_{p}\left(t_{j+1}\right)=\mathbf{0} \text {, }
$$

where the material parameters entering Eq. (38) to get matrix $\widetilde{\mathbf{P}}\left(t_{j+1}\right)$ are evaluated under the same assumptions as in Section 3.1.

We usually require the material parameters, including the coefficient of permeability, to be constant within a given element. Eqs. (7) and (10) suggest that the volumetric strain is constant as well. When assuming a linear approximation of the pore pressure, the vector of nodal pore pressure values of the $i^{\text {th }}$ element becomes

$$
\left(d_{i, p}\right)^{\mathrm{T}}=\left\{d_{p 1}, d_{p 2}\right\}_{i} .
$$

The constant effective mean stress $\sigma_{i, m}^{\text {eff }}$ in the $i^{\text {th }}$ element is then given by

$$
\sigma_{i, m}^{\text {eff }}=\sigma_{m}-\frac{\left(d_{p 1}+d_{p 2}\right)_{i}}{2}
$$

For the sake of consistency, the volumetric strain $\epsilon_{v}$ and the water pore pressure $p^{w}$ should be of the same order (recall Eq. (8)). Here, the desirable consistency of the solution is achieved by applying so-called selective integration to integrate the compressibility matrix (one-point Gauss' quadrature).

Once the discretization in space has been performed Eq (40) represents a set of ordinary differential equations in time, which can be integrated numerically, [2]. To proceed, consider again the generalized trapezoidal rule such that

$\dot{d}_{p}\left(t_{j+1}\right)=\frac{1}{\tau \Delta t}\left(d_{p}\left(t_{j+1}\right)-\widetilde{d}_{p}\left(t_{j+1}\right)\right)$,

$\widetilde{d}_{p}\left(t_{j+1}\right)=d_{p}\left(t_{j}\right)+(1-\tau) \Delta t \dot{d}_{p}\left(t_{j}\right)$.

Substitution of Eq. (43) into Eq. (40) finally leads to a system of linear algebraic equations given by

$\frac{1}{\tau \Delta t}\left(\mathbf{C}+\tau \Delta t \widetilde{\mathbf{P}}\left(t_{j+1}\right)\right) d_{p}\left(t_{j+1}\right)=\frac{1}{\tau \Delta t} \mathbf{C} \widetilde{\boldsymbol{d}}_{p}\left(t_{j+1}\right)$.

The initial condition prescribing the water pore pressure at the beginning of loading, $t=0$ takes the form

$d_{i, p}=d_{i, p}(0)=-\sigma_{m}+\sigma_{i, m}^{\text {eff }}(0), \quad i=1, \ldots, n$,

where $n$ is the number of elements.

\section{Numerical results}

Before proceeding with the results derived from the presented formulation we draw the readers attention to Fig. 6, which manifests several drawbacks associated with the solution of the consolidation problem when applied to the standard Cam clay model. The solid line shows results derived experimentally. The theoretical predictions were derived assuming the material parameters listed in Table 1. The dash-dotted line follows from numerical calculations assuming the linear consolidation line (virgin soil) and the deformation dependent coefficient of permeability, while the dashed line was found from the bilinear consolidation line, but keeping the coefficient of permeability constant. Clearly, neither the deformation dependent variation of the coefficient of

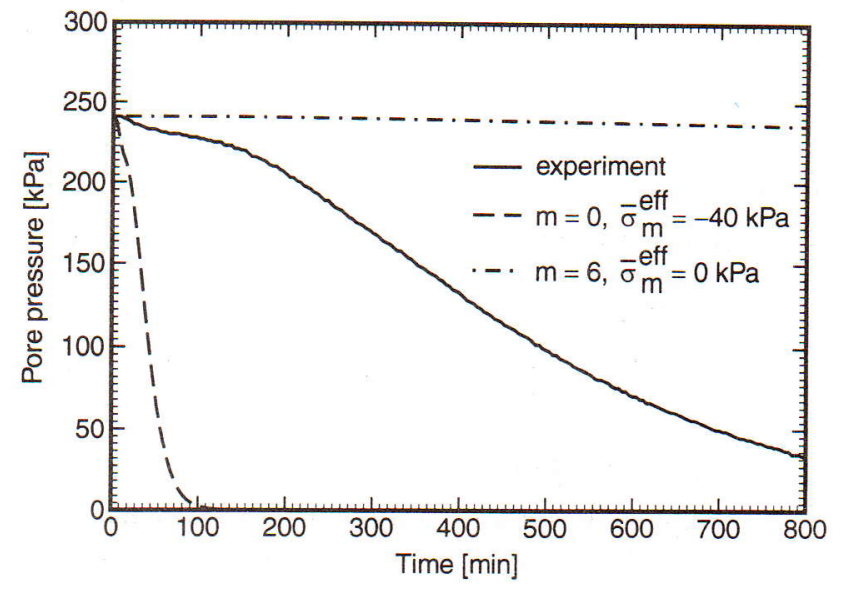

Fig. 6: Time variation of pore pressure

Table 1: Material parameters

\begin{tabular}{|c|c|c|c|c|c|}
\hline$\kappa[\mathrm{kPa}]$ & $\lambda[\mathrm{kPa}]$ & $e_{0}$ & $K_{0}[\mathrm{~m} / \mathrm{s}]$ & $\bar{\sigma}_{m}^{\text {eff }}[\mathrm{kPa}]$ & $m$ \\
\hline 0.01 & 0.1 & 0.5 & $5.010^{9}$ & -40 & 6 \\
\hline
\end{tabular}

permeability nor the bilinear form of the consolidation line can itself describe the true consolidation process and therefore both of them should be taken into consideration when simulating transport of water in soft soils.

In a nutshell, the first assumption essentially labels soils as being normally consolidated from their virgin state, which further contributes to a rather slow initial phase of the process of consolidation, Fig. 6 (dash-dotted line). In their natural state, however, soils are usually in overconsolidated states, due to unloading from the original stress state (e.g., water table fluctuation). To account for the prior loading history we introduce the influence zone variation as a function of a certain level of the effective mean stress, usually referred to as structural strength. This level of the effective mean stress can be identified with preconsolidation pressure. In the present formulation, the structural strength is introduced through a bilinear shape of the consolidation line [8], [1]. The effect of this step becomes evident when examining Eq. (12). In particular, a lower value of $\hat{\lambda}$ at the initial stage increases the coefficient of permeability, leading to an acceleration of the consolidation process at this stage, a phenomenon observed in the experiments. 
The second shortcoming comes from the originally assumed constant value of the coefficient of permeability, responsible for an abrupt increase in the rate of consolidation as the effective mean stress developed during consolidation becomes appreciable, Fig. 6 (dashed line). However, this stage of consolidation is associated with a significant deformation of the skeleton, leading to a decrease in the volume of the pores and subsequently to an decrease in the coefficient of permeability. Based on our extensive numerical investigations of isotropic consolidation [8], we proposed an exponential relation between the coefficient of permeability and the actual void ratio represented by Eq. (10). Note that this relationship was suggested by the authors based solely on numerical results. Nevertheless, it conforms with an experimental observation, [9] pp. 138, fairly well.

This set of numerical experiments also served to examine the individual numerical techniques. The results were rather inconclusive as to what method should be preferred, since all methods performed equally well in this very specific consolidation problem. Thus the fact that the finite volume method is usually more stable ruled the selection of this method for the subsequent numerical program, which provides a qualitative description of the above mentioned requirements.

First, the influence of the bilinear consolidation line Fig. 1, is investigated. The location of the slope discontinuity on the $\kappa-\lambda$ line corresponds to a given value of the structural strength of the soil. This value can be estimated using, e.g., the Casagrande method [3]. The results for various values of the structural strength $\bar{\sigma}_{m}^{\text {eff }}$ are plotted in Fig. 7, assuming $m$ equal to 6 . It is evident that increasing the magnitude of $\bar{\sigma}_{m}^{\text {eff }}$ speeds up the rate of consolidation in the first stage quite substantially.

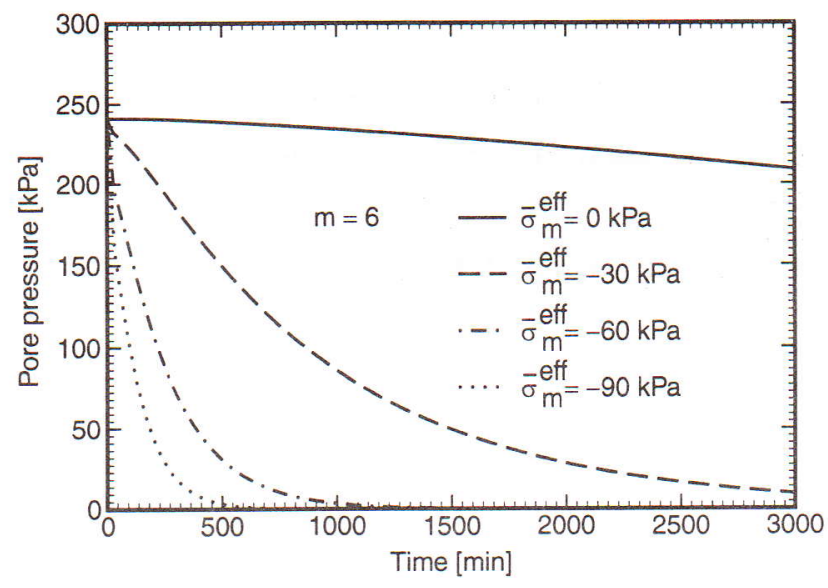

Fig 7: Time variation of pore pressure

The second objective aims at slowing down the consolidation process in the second stage of consolidation associated with a decreasing volume of pores due to skeleton deformation. This phenomenon is taken into account by incorporating Eq. (10) into the numerical procedure. Results for various values of exponent $m$ and for a given structural strength $\bar{\sigma}_{m}^{\text {eff }}=-30 \mathrm{kPa}$ are presented in Fig. 8. The dash-dotted line shows a rather gradual decrease in the pore pressure profile with increasing time, which is in better agreement with experimental observations.

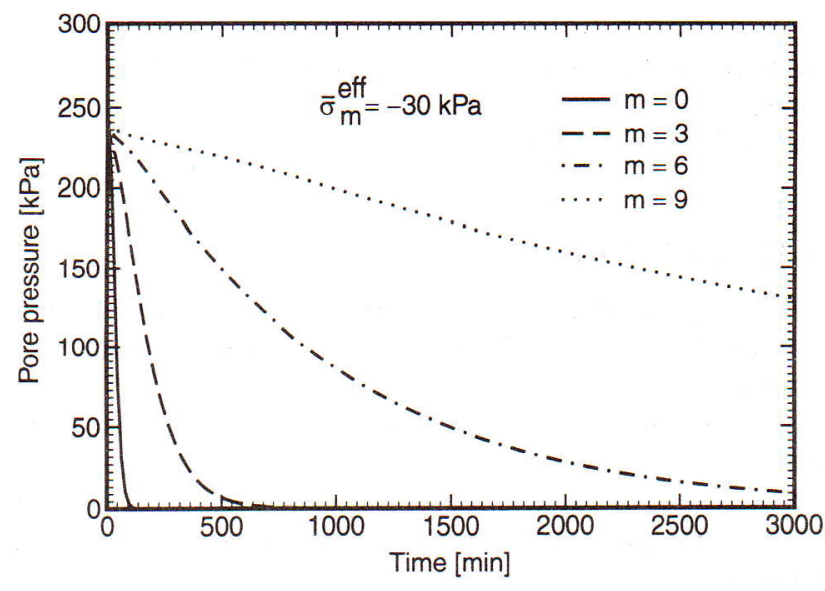

Fig. 8: Time variation of pore pressure

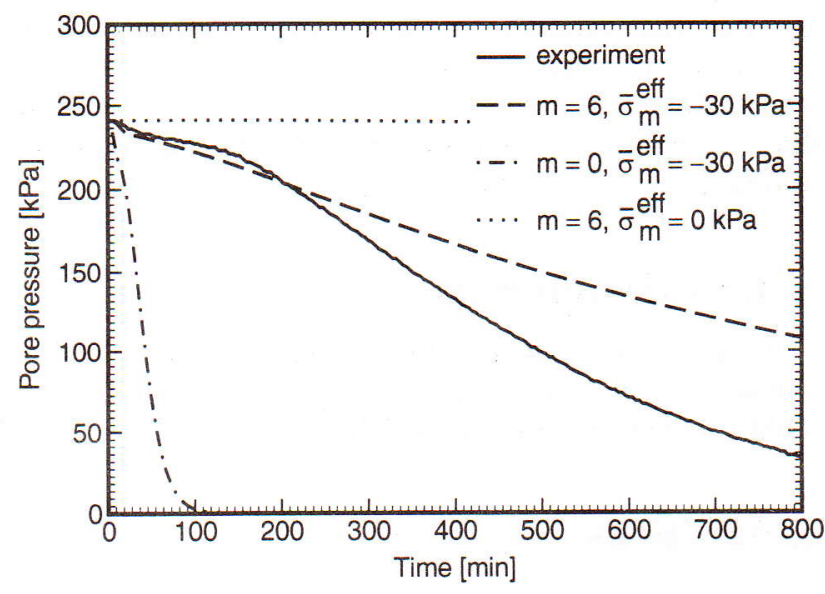

Fig. 9: Time variation of pore pressure

Fig. 9 then summarizes the present theory. First, recall that the dotted line was obtained without taking into account the bilinear consolidation line. In such a case, a rather high value of $m$ locks up the numerical procedure at the beginning of consolidation. The dashed line then re ects incorporation of both requirements. Up to now a simple trial and error procedure was employed to derive optimal values of $m$ and $\bar{\sigma}_{m}^{\text {eff }}$.

Furthermore, reproducing laboratory data requires that we supply, apart from the structural strength parameters $\left(\bar{\sigma}_{m}^{\text {eff }}, m\right)$, the following material parameters: the initial void ratio $\bar{e}_{0}$, the initial coefficient of permeability $K_{0}$, the swelling index $\kappa$ and the compression index $\lambda$. The last two material parameters and the starting value of preconsolidation pressure could be specified from the steady state response corresponding to the diagram shown in Fig. 1. This, however, would require carrying out a number of isotropic consolidation tests for several predefined levels of isotropic pressures attained at the end of the consolidation process, while measuring at the same time the change in volumetric strain $\Delta \epsilon_{v}$. Such an approach would become not only time consuming but also burdened by an additional experimental error associated with the difficulty of measuring $\Delta \epsilon_{v}$. Also, the determination of $\bar{e}_{0}$ and $K_{0}$ would require additional laboratory tests. A simple solution to this problem, however, is offered by following the steps of mixed experimental and numerical methods. In such a case, combining the experi- 
mental measurements and numerical computations in a suitable optimization environment provides an efficient tool for inferring the desired parameters from a single laboratory test. This issue is discussed in [10].

\section{Conclusions}

The present paper was concerned with an effect of structural strength in the computational model of isotropic consolidation in which the skeleton deformation is governed by the modified Cam clay model. Several numerical techniques to solve the isotropic consolidation problem were explored. The finite volume method seems to perform best in the present problem of one dimensional isotropic consolidation.

Both the numerical results and the experimental data proved the need to incorporate the structural strength of soils and the time variation of the coefficient of permeability into the computational model. An independent experimental observation confirmed the exponential form of the relationship between the coefficient of permeability and the current void ratio proposed by the authors.

\section{Acknowledgments}

Financial support for this project was provided by Ministry of Education projects No. MSM:210000001 and MSM:210000003, and by GAČR 103/02/0688/A.

\section{References}

[1] Asaoka, A., Nakano, M., Noda, T.: Super loading yield surface concept for the saturated structured soils. NUMGE 98, edited by A. Cividini, Udine (Italy), 1998, Vol. 14, p. 233-242.

[2] Bittnar, Z., Šejnoha, J.: Numerical methods in structural mechanics. New York: ASCE Press, 1996.

[3] Bowles, J. E.: Foundation analysis and design. New York: McGraw-Hill, 1996.
[4] Dluzewski, J. M.: Large strain consolidation for elasto-plastic soils. NUMGE 98, edited by A. Cividini, Udine (Italy): 1998 , p. $473-482$.

[5] Hughes, T. J. R.: The Finite Element Method, Linear Static and Dynamic Finite Element Analysis. New Jersey: Prentice Hall, Inc., 1987.

[6] Krejčí, T., Nový, T., Sehnoutek L., Šejnoha, J.: Structure -subsoil interaction in view of transport processes in porous media. CTU Reports, Prague, 2001, Vol. 5, p. 1-81.

[7] Kuklík, P., Mareš, J., Šejnoha, M.: The structural strength of soil from the isotropic consolidation point of view. APCOM 99, edited by C. M. Wang, K. H. Lee, K. K. Ang, Singapore, 1999, Vol. 2, p. 797-802.

[8] Kuklík, P., Mareš, J., Šejnoha, M.: Evaluation of the modified cam clay model with reference to isotropic consolidation. CTU Reports, Prague, 1999, Vol. 3, p. 47-54.

[9] Lewis, R. W., Schrefler, B. A.: The finite element method in static and dynamic deformation and consolidation of porous media. Chichester (New York): Wiley, 1998.

[10] Janda, T., Kuklík, P., Šejnoha, M: Mixed experimental and numerical approach to evaluation of material parameters of clayey soils in a review for publication in International Journal of Geomechanics.

Doc. Ing. Milan Šejnoha, Ph.D.

phone: +420224354494

fax: +420224310775

e-mail: sejnom@fsv.cvut.cz

Doc. Ing. Pavel Kuklík, CSc.

phone: +420224354486

kuklikpa@fsv.cvut.cz

Tomáš Janda

Department of Structural Mechanics

Czech Technical University in Prague

Faculty of Civil Engineering

Thákurova 7 ,

16629 Prague 6, Czech Republic 